\title{
Charge Transfer at Hybrid Organic-Inorganic Interface
}

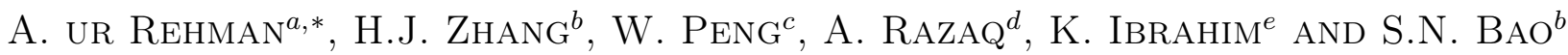 \\ ${ }^{a}$ Physics Department, University of Agriculture, Faisalabad 38040, Pakistan \\ ${ }^{b}$ Physics Department, Zhejiang University, Hangzhou 310027, P.R. China \\ ${ }^{c}$ Physics Department, Shandong University of Science and Technology, Shandong, 266510, P.R. China \\ ${ }^{d}$ Physics Department, COMSATS Institute of Information Technology, Lahore, Pakistan \\ ${ }^{e}$ Institute of High Energy Physics, Chinese Academy of Sciences, P.R. China
}

\begin{abstract}
Iron phthalocyanine molecule $(\mathrm{FePc})$ thin films were deposited on $\mathrm{Cu}(100)$ and $\mathrm{Si}(100)$ substrates in sequential steps and the interface was characterized by means of scanning tunneling microscope, X-ray photoemission spectroscopy, and its sister technique ultra violet photoemission spectroscopy. At room temperature disordered structures are observed. However at elevated temperature $\approx 480^{\circ} \mathrm{C}$, a long range ordered pattern is noticed indicating the increase of diffusion of adsorbed molecules on the substrate surface. All the photoemission spectra exhibited a clear change and shift in the adsorbed peaks. The change in binding energy of $\mathrm{C} 1 s$ core level as revealed by X-ray photoemission spectroscopy is $V_{b}=0.18 \mathrm{eV}$. Our calculated work function of the clean Si (100) surface is $4.09 \mathrm{eV}$. The hole and electron injection barriers are estimated as $1.22 \mathrm{eV}$ and $-1.38 \mathrm{eV}$, respectively. Based on the measured quantities, the interface dipole potential is found to be $-0.04 \mathrm{eV}$. A minute charge transferred from each adsorbed molecule to the substrate is estimated as $c a .8 .565 \times 10^{-22} \mathrm{C}$. Besides, our computational density functional theory findings revealed a strong adsorbate-substrate interaction.
\end{abstract}

DOI: 10.12693/APhysPolA.134.434

PACS/topics: FePc, Si (100), Cu(100), STM, PES, nanostructures, work function, charge density, interface dipole

\section{Introduction}

Phthalocyanine $(\mathrm{Pc})$ molecules have gained tremendous attention due to their widespread applications (e.g. solar cells [1], photodynamic cancer therapy (PDT) [2], gas-sensing devices [3]). Due to their excellent electronic and optical properties they have been perceived as promising building blocks for nanotechnology [4]. In this connection, the interfaces between organic films and metals/inorganic semiconductors play a decisive role. The fabrications of organized thin films of Pcs have been playing an important role in incorporating Pcs into devices [5]. That is why the growth and morphology of metal phthalocyanine thin films have largely been studied on dielectric and conducting surfaces at different molecular coverages [6]. It is well known that the optical, electrical, thermal, and magnetic properties of molecular materials are intimately related to their highly ordered structures. Therefore, understanding and controlling the organization of organic molecules on electrode surfaces at the molecular level is a very important prerequisite to improve the performance of metal phthalocyanine (MPc) based organic electronic devices. Keeping in view the importance of Pc family, a less addressed molecule, i.e. FePc on $\mathrm{Cu}(100)$ and $\mathrm{Si}(100)$ surfaces was studied by means of scanning tunneling microscopy (STM) and photoemission spectroscopy (PES). The geometric and structural transitions were further explained with the help of density functional theory calculations (DFT). The chemical structure of Fe-Pc molecule is shown in Fig. 1.

\footnotetext{
*corresponding author; e-mail: ateeq215@gmail.com
}

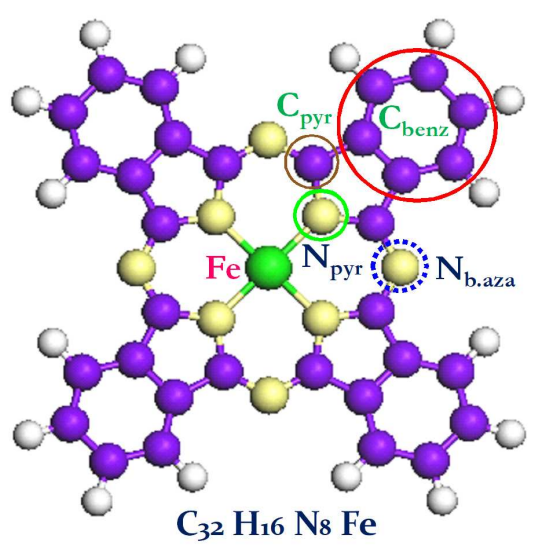

Fig. 1. Top view of the molecular structure of FePc.

\section{Experimental and calculation details}

The experiments were conducted in a ultrahighvacuum (UHV) VT-SPM system (Omicron) with the base pressure better than $8 \times 10^{-10}$ mbar. Before depositing organic material, substrate surfaces were cleaned by standard cleaning procedures. Commercial FePc powder (Sigma-Aldrich) was carefully degassed at $450 \mathrm{~K}$ overnight for purification. Molecules were then thermally deposited from a home-made tantalum boat at an evaporation temperature of about $750 \mathrm{~K}$, with the substrates held at room temperature (RT). The surface structural characterization was performed by STM during which the substrate was kept at RT. STM images were recorded in constant-current mode and processed using WSxM software [7]. The interfacial electronic structure was studied with PES. The spectroscopic measurements were carried 
out with unpolarized $\mathrm{He}(\mathrm{I})(21.2 \mathrm{eV})$ and a monochromatic $\mathrm{Mg} K_{\alpha}(1253.6 \mathrm{eV})$ radiation source. DFT calculations (using DMol3 package) were used for the computational calculations [8].

\section{Results and discussion}

A multilayer STM image of $\mathrm{FePc}$ on $\mathrm{Cu}(100)$, Fig. 2a, reveals random adsorption of molecules. However, still individual molecules are discernible. Monolayer STM image after annealing the substrate at $\approx 435 \mathrm{~K}$ is shown in Fig. 2b. In this case, formation of large and ordered molecular arrangement with oblique unit cell (yellow color) is observable, which increases the packing density of molecules. Each molecule appears as a crossed four-lobe structure with a protrusion at the center. This ordered assembling is a result of the strong intermolecular interaction. Central bright spot appearance and the four lobes show flat lying adsorption [9].
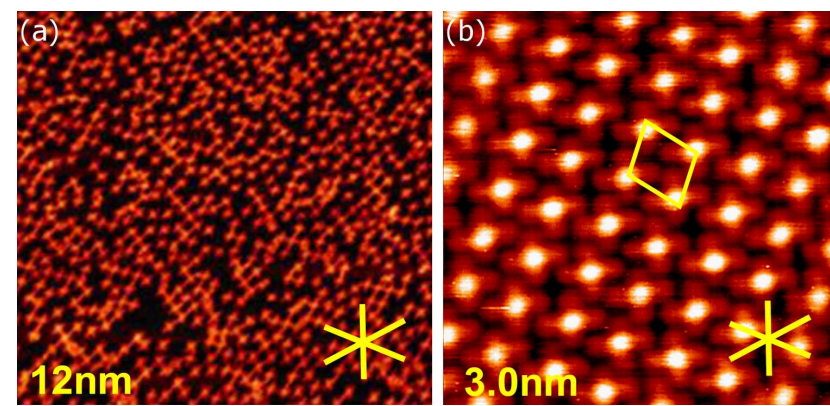

Fig. 2. (a) STM graph of $5 \mathrm{ML}$ coverage of $\mathrm{FePc}$ on $\mathrm{Cu}(100)$. The image $\left(60 \times 60 \mathrm{~nm}^{2}\right)$ was taken at RT with $V=-3.2 \mathrm{~V}$, and $I=0.08 \mathrm{nA}$. (b) STM image $(15 \mathrm{~nm} \times 15 \mathrm{~nm})$ of $1 \mathrm{ML} \mathrm{FePc}$ on the substrate. The close packed directions of $\mathrm{Cu}(100)$ are represented by yellow stars. Unit cell of the adsorbate is shown with yellow color.

Moreover, molecular central bright spot is ascribed to the partially filled $d$-orbital of iron (Fe) [10] and four lobes could be assigned to the four iso-indole units of the molecules [11]. The adsorption configuration has great influence on the performance of organic molecular based devices [12]. Keeping in view the importance of adsorption geometry, FePc molecule was adsorbed on $7 \times 7$ unit cell of $\mathrm{Cu}(100)$ as shown in Fig. 3. The molecule was optimized and an inspection of Fig. 3a,b discloses the rotation and transformation of the molecule.

The molecular plane of $\mathrm{FePc}$ is horizontally lying on the substrate surface. However, the molecular plane is bent with an angle of about $12^{\circ}$, as a result of strong molecule-substrate interaction. Moreover, major molecular axis is rotated relative to the [01-1] crystallographic direction by an angle of $5^{\circ}$. Molecular phase transformation has often been observed for metal phthalocyanines on metal surfaces $[13,14]$. The calculated density of states (DOS) of the adsorbed and free FePc molecule are shown in Fig. 3c. Figure 4 represents the valence level spectra of the sequential deposition of $\mathrm{FePc}$ on the
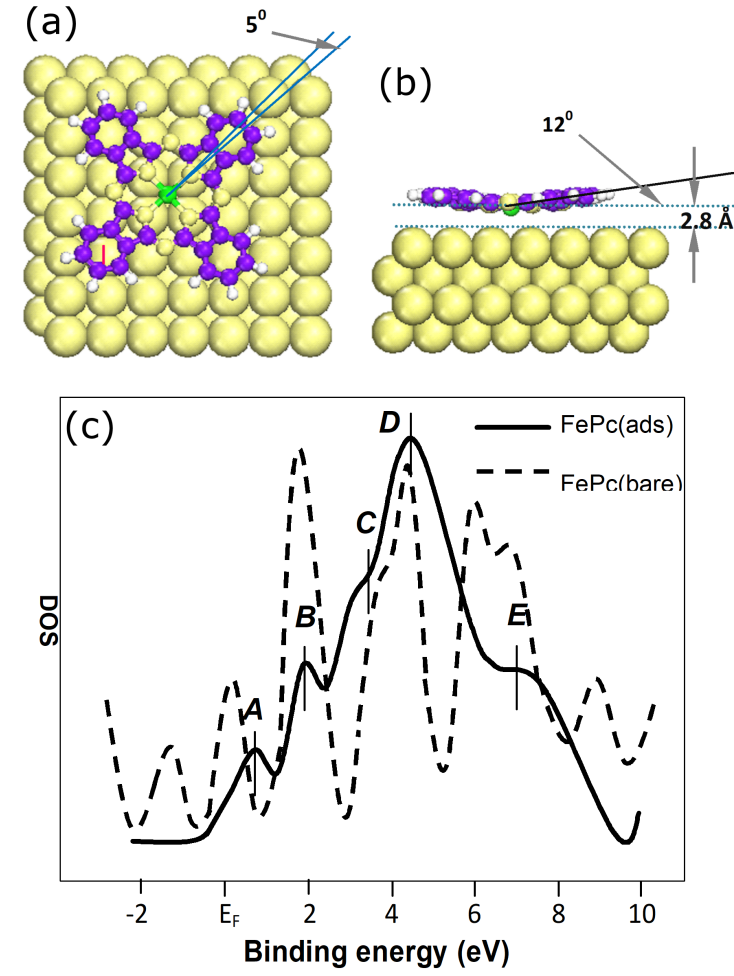

Fig. 3. Top (a) and side (b) views of adsorption geometry of molecule on $\mathrm{Cu}(100)$ substrate, (c) calculated DOS of a FePc molecule isolated and adsorbed. substrate. This figure consists of three parts: Fig. 4a - the HOMO and the TOP of HOMO as a function of FePc thickness, Fig. 4b - the valence band (VB) structures, and Fig. $4 \mathrm{c}$ - the high binding energy cutoffs of the secondary electrons. A bottom spectrum, Fig. 4b, purely corresponds to the substrate signal. No adsorbate related feature is observed. However, after ML coverage, coverage dependent growth of $\alpha, \beta, \delta$ and $\gamma$ from the organic molecules can easily be observed. At $5 \mathrm{ML}$, only these organic features are visible.

At $10 \mathrm{ML}$, organic thin film related features are fully grown. For 1.0 ML FePc coverage, PE features are located at 1.64, 3.89, 7.05, and $10.0 \mathrm{eV}$ below the Fermi level, respectively. These features remain almost stagnate until the molecular coverage reaches 5.0 ML. At the termination coverage, a shift in these features is visible. Also shape of the features is not more the same. Both shifts and the perturbed shapes of the organic features suggest the charging of the films. During the coverage $1 \mathrm{ML}$ to $10 \mathrm{ML}$, HOMO shifts from 1.65 to $1.77 \mathrm{eV}$. The overall shift in HOMO is $\approx 0.12 \mathrm{eV}$, and it represents charge transfer from the adsorbate to the substrate. A visible change in the VB features and TOP of HOMO is a result of adsorbate-substrate interaction. The Si substrate does not have much influence on the thick organic layers. Therefore, the shift in BE with varying molecular coverage represents adsorbate-substrate interaction. The cutoff energies, Fig. 4c, related to 0.0, 1.0€Ź5.0 and 10.0 


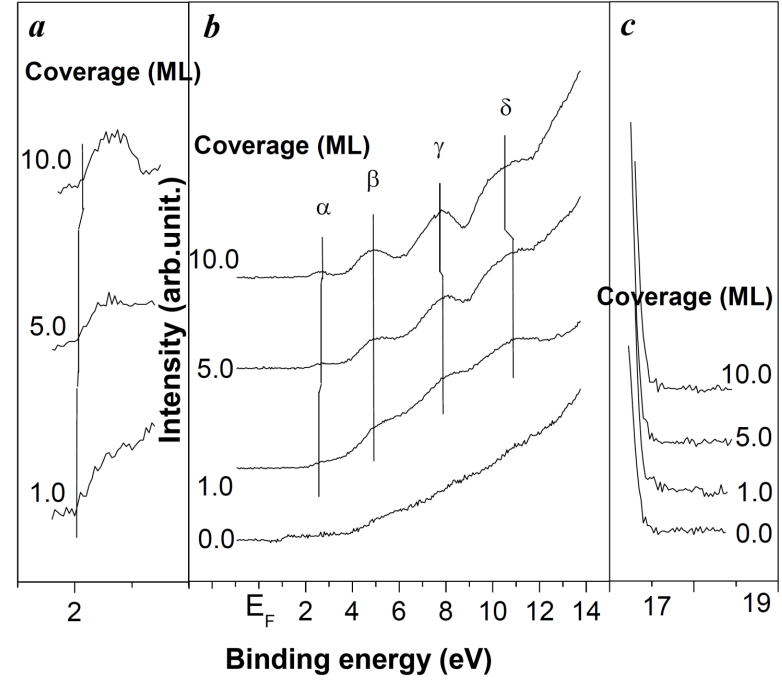

Fig. 4. UP spectra of FePc on $\mathrm{Si}(100)$ : (a) HOMO and the TOP of HOMO as a function of FePc thickness, (b) the valence band structures, (c) the cutoff of the secondary electrons.

ML coverage are $16.89,17.18,16.99$ and $16.97 \mathrm{eV}$, respectively. The work functions calculated with the help of cut off energies with the basic equation $\varphi=h \nu-\left(E_{c u t}-E_{F}\right)$ are $4.08,4.12,4.18$, and $4.22 \mathrm{eV}$. The lowest work function value, i.e. $4.08 \mathrm{eV}$ corresponds to the bare substrate. Upon deposition, work function increases and finally switches to $4.22 \mathrm{eV}$, i.e. the work function of the thin organic film. Work function modification of overlayer Pc molecular adsorption is a result of the formation of interface dipole potential [15]. The core level (CL) features at increasing amount of molecular coverage are shown in Fig. 5. With the stepwise deposition of the organic material the intensity of the $\mathrm{C} 1 \mathrm{~s}$ core level increases and it reaches to its maximum level with the terminal coverage, as shown in Fig. 5a.

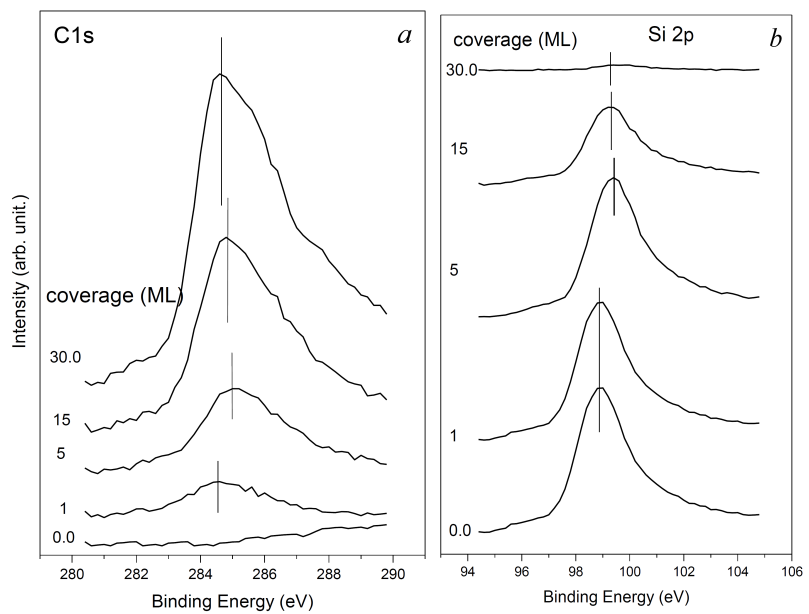

Fig. 5. Core level PE spectra of FePc on Si (100): (a) C $1 s$ core level, (b) Si $2 p$ core level.

Contrary to this, with each deposition step intensity of the substrate signal decreases and peak shifts towards the higher binding energy as shown in Fig. 5b. At 1.0 ML, C $1 s$ peak attributed to organic molecule is grown. With further increase in the organic coverage, Pc derived $\mathrm{C} 1 \mathrm{~s}$ CL shifts towards/away from its original position and increases in intensity. A total shift in the CL features to the higher binding energy is $0.18 \mathrm{eV}$. At $30 \mathrm{ML}$ coverage, surface is completely covered by the organic material. A critical analysis of this shifting on the one hand connotes the modification of the molecular electronic structure, due to the strong interaction at the interface, and on the other hand it indicates charge transfer from the planar $\mathrm{FePc}$ molecule to the substrate $[15,16]$. By taking into account CL findings, the charge transfer from each adsorbed molecule to the substrate is found $8.565 \times 10^{-22} \mathrm{C}$. With the well known surface area of FePc molecule, $2.19 \mathrm{~nm}^{2}$, the charge density is estimated as $2.0 \times 10^{-39} \mathrm{C}^{2} / \mathrm{nm}^{2}$. As a result of charge transfer, a dipole is formed at the interface. The width of the dipole is $c a . d=1.32 \AA$. The change in binding energy of $\mathrm{C} 1 s$ is $V_{b}=0.18 \mathrm{eV}$. The hole injection barrier $\left(\Phi_{b h}=\right.$ TOP of HOMO $\left.-V_{b}\right)$ obtained with and without considering $V_{b}$ are estimated 1.22 and $1.42 \mathrm{eV}$, respectively. By using the previously determined HOMO and LUMO gap [17], the electron injection barrier is $\Phi_{b e}=\Phi_{b h}-E_{g}=1.22-1.38=-0.11 \mathrm{eV}$. Ionization potential, the distance between the vacuum level and the TOP of HOMO, is calculated by: IP = $e \Phi_{F e P c}+$ TOP of HOMO $=(4.22+1.65)=5.87 \mathrm{eV}$. This value is in agreement with the previously calculated value [17]. The interface dipole potential is found to be $e D=\Delta \Phi-V_{b}=0.1-0.18=-0.08 \mathrm{eV}$. The small value and negative sign of $e D$ suggests that very small charge is transferred from FePc molecule to the $\mathrm{Si}$ (100) sample surface.

\section{Conclusion}

A study on the interfaces between iron phthalocyanine molecule deposited on $\mathrm{Cu}(100)$ and $\mathrm{Si}(100)$ surfaces have been carried out by means of STM and PES. Our STM findings show that at low coverage no ordered structures are observable. However, at an elevated temperature long range ordered molecular arrangement is observed. The PES data show clear change and shift in the adsorbate associated peaks. The hole and electron injection barriers are estimated as $1.22 \mathrm{eV}$ and $-1.38 \mathrm{eV}$, respectively. Based on the fundamental quantities, the interface dipole potential is found to be $-0.08 \mathrm{eV}$. A nominal charge transferred from each adsorbed molecule to the substrate is estimated as $8.565 \times 10^{-22} \mathrm{C}$. Moreover, our computational findings revealed a strong moleculesubstrate interaction which severely distorts the shape of the molecule.

\section{Acknowledgments}

This work was supported by the Startup Research Grant Project (SRGP \#518) from the Higher Education Commission of Pakistan. 


\section{References}

[1] K.S. Yook, B.D. Chin, J.Y. Lee, B.E. Lassiter, S.R. Forrest, Appl. Phys. Lett. 99, 043308 (2011).

[2] J.D. Spikes, Photochem. Photobiol. 43, 691 (1986).

[3] X. Lu, K.W. Hipps, X.D. Wang, U.J. Mazur, Am. Chem. Soc. 118, 7197 (1996).

[4] G. de La Torre, C.G. Claessens, T. Torres, Chem. Commun. 28, 2000 (2007).

[5] J.P. Bourgoin, F. Doublet, S. Palacin, M. Vandevyver, Langmuir 12, 6473 (1996).

[6] C. Iacovita, M.V. Rastei, B.W. Heinrich, T. Brumme, J. Kortus, L. Limot, J.P. Buche, Phys. Rev. Lett. 101, 116602 (2008).

[7] I. Horcas, R. Fernandez, J.M.G. Rodriguez, J.G. Herrero, A.M. Baro, Rev. Sci. Instrum. 78, 13705 (2007).

[8] B. Delley, J. Chem. Phys. 113, 7756 (2000).

[9] L. Gao, W. Ji, Y.B. Hu, Z.H. Cheng, Z.T. Deng, Q. Liu, N. Jiang, X. Lin, W. Guo, S.X. Du, W.A. Hofer, X.C. Xie, H.-J. Gao, Phys. Rev. Lett. 99, 106402 (2007).
[10] X. Lu, K.W. Hipps, J. Phys. Chem. B 101, 5391 (1997).

[11] S.C. Bobaru, E. Salomon, J.-M. Layet, T. Angot, J. Phys. Chem. C 115, 5875 (2011).

[12] M.C. Cottin, J. Schaffert, A. Sonntag, H. Karacuban, R. Möller, C.A. Bobisch, Appl. Surf. Sci. 258, 2196 (2012).

[13] M. Casarin, M. Di Marino, D. Forrer, M. Sambi, F. Sedona, E. Tondello, A. Vittadini, V. Barone, M. Pavone, J. Phys. Chem. C 114, 2144 (2010).

[14] S.H. Chang, S. Kuck, J. Brede, L. Lichtenstein, G. Hoffmann, R. Wiesendanger, Phys. Rev. B 78, 233409 (2008).

[15] J.X. Tang, K.M. Lau, C.S. Lee, S.T. Lee, Appl. Phys. Lett. 88, 232103 (2006).

[16] F. Petraki, H. Peisert, I. Biswas, T. Chasse, J. Phys. Chem. C 114, 17638 (2010).

[17] M.S. Liao, S. Scheiner, J. Chem. Phys. 114, 9780 (2001). 